SAÚDE E AMBIENTE

V.8 $\cdot$ N.1 Agosto/Setembro/Outubro - 2019

ISSN Digital: 2316-3798

ISSN Impresso: 2316-3313

DOI: 10.17564/2316-3798.2019v8n1p43-60 ORCID: 0000000000000

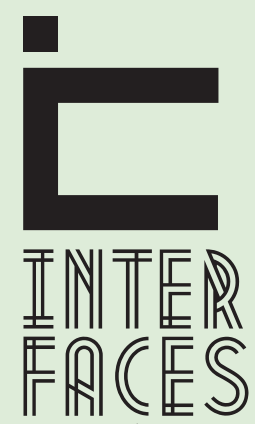

CIENTÍFICAS

\section{CARACTERÍSTICAS CLÍNICO EPIDEMIOLÓGICAS DOS CASOS DE ACIDENTES COM ESCORPIÕES NOS TERRITÓRIOS DE SERGIPE, BRASIL' ${ }^{1}$}

\section{CLINICAL EPIDEMIOLOGICAL CHARACTERISTICS OF CASES OF ACCIDENTS WITH SCORPIONS IN THE TERRITORIES OF SERGIPE, BRAZIL}

CARACTERIISTICAS CLIINICAS EPIDEMIOLÓGICAS DE LOS CASOS DE ACCIDENTES COM ESCORPIONES EN LOS TERRITORIOS DE SERGIPE, BRASIL

Carlos Adriano de Oliveira Almeida ${ }^{2}$ Guilherme Mota da Silva ${ }^{3}$ Andressa Sales Coelho ${ }^{4}$

1 Artigo baseado na dissertação de Mestrado de Carlos Adriano de Oliveira Almeida, intitulada "Acidentes Escorpiônicos no Estado de Sergipe: Análise Espaço-Temporal de 2004 a 2014”, defendida em 2016 no Programa de Pós-Graduação em Saúde e Ambiente da Universidade Tiradentes.

\section{RESUMO}

Este trabalho teve por objetivo identificar as características clínico-epidemiológicas dos casos de acidentes com escorpiões no estado de Sergipe. Trata-se de uma análise descritiva, realizada a partir de registros dos acidentes com animais peçonhentos relacionados à envenenamento de escorpião do Sistema de Informação sobre Doenças Notificáveis (SINAN - Sistema de Informação de Agravos de Notificação), no período entre 2004 a 2014. Foram notificados, no período proposto, 5.133 casos de acidentes por escorpião. Destes, ocorreram quatro óbitos no período estudado, sendo $75,0 \%$ deles em crianças e adolescentes; a faixa etária mais acometida em Sergipe foi entre 20 e 29 anos 10,3\% e constatou-se que o sexo feminino foi o mais acometido com 57,1\%. Quanto aos dados clínico-epidemiológicos tiveram como localização anatômico do corpo mais atingida, membros inferiores $37,1 \%$. Já na classificação de gravidade dos casos, encontrou-se a maioria foram diagnosticados clinicamente como leves $84,4 \%$. Em relação a busca pelo atendimento médico após os acidentes escorpiônicos, 56,0 \% das vítimas buscaram o atendimento em até 3 horas, com diferenças significativas na distribuição entre os territórios ( $p<0,0001)$. As manifestações locais mais frequentes nos casos de acidentes no período estudado foram dor, edema e eritema. Além disso, as manifestações sistêmicas mais frequentes foram as vagais, hemorrágicas e miolíticas com diferenças significativas na distribuição entre os territórios ( $P$ $<0,05)$. Diante deste cenário é necessário programar estratégias de educação em saúde capazes de controlar ou reduzir os fatores de risco de acidentes escorpiônicos da população. 


\section{PALAVRAS CHAVES}

Escorpiões. Sergipe. Epidemiologia.

\section{ABSTRACT}

This study aimed to identify the clinical and epidemiological characteristics of cases of scorpion accidents in the state of Sergipe. This is a descriptive analysis, based on records of occurrences with venomous animals related to poisoning of the Escapade Information System (SINAN) notifications, from 2004 to 2014.There have been reports, in the proposed period, 5.133 cases of accidents per scorpion. Of these, four deaths occurred in the period studied, $75.0 \%$ of them in children and adolescents; the most affected age group in Sergipe was between 20 and 29 years $10.3 \%$ and it was found that the female was the most affected with $57.1 \%$. As for the clinical-epidemiological data, the anatomical location of the most affected body was $37.1 \%$. Already in the severity classification of the cases, it was found the majority were diagnosed clinically as mild $84.4 \%$. Regarding the search for medical care after the scorpionic accidents, $56.0 \%$ of the victims sought care within 3 hours, with significant differences in the distribution among the territories $(p<0.0001)$. The most frequent local manifestations in cases of accidents in the studied period were pain, edema and erythema. In addition, the most frequent systemic manifestations were vagal, hemorrhagic and myolitic with significant differences in the distribution between territories $(P<0.05)$. In view of this scenario, it is necessary to plan health education strategies capable of controlling or reducing the scorpion risk factors of the population.

\section{KEYWORDS}

Scorpions. Sergipe. Epidemiology.

\section{RESUMEN}

Este trabajo tuvo por objetivo identificar las características clínico-epidemiológicas de los casos de accidentes con escorpiones en el estado de Sergipe. Se trata de un estudio descriptivo y retrospectivo utilizando un enfoque cuantitativo basado en datos secundarios de registros relacionados con la envenenación de escorpión del Sistema de Información sobre Enfermedades notificables (SEMAN - Sistema de Información de Agravios de Notificación) de 2004 a 2014. Se notificaron, en el período propuesto, 5.133 casos de accidentes por escorpión. De estos, ocurrieron cuatro muertes en el período estudiado, siendo el 75,0\% de ellos en niños y adolescentes; el grupo de edad más acometido en Sergipe fue entre 20 y 29 años 10,3\% y se constató que 
el sexo femenino fue el más acometido con el 57,1\%. En cuanto a los datos clínico-epidemiológicos tuvieron como localización anatómica del cuerpo más afectada, miembros inferiores 37,1\%. En la clasificación de gravedad de los casos, se encontró la mayoría fueron diagnosticados clínicamente como leves 84,4\%. En cuanto a la búsqueda por la atención médica después de los accidentes escorpiónicos, el 56,0\% de las víctimas buscaron la atención en hasta 3 horas, con diferencias significativas en la distribución entre los territorios $(p<0,0001)$. Las manifestaciones locales más frecuentes en los casos de accidentes en el período estudiado fueron dolor, edema y eritema. Además, las manifestaciones sistémicas más frecuentes fueron las vagales, hemorrágicas y miolíticas con diferencias significativas en la distribución entre los territorios ( $P$ $<0,05)$. Ante este escenario es necesario programar estrategias de educación en salud capaces de controlar o reducir los factores de riesgo de accidentes escorpiónicos de la población.

\section{PALABRAS CLAVES}

Escorpiones. Sergipe. Epidemiología.

\section{INTRODUÇ̃̃̃O}

Os escorpiões são animais terrestres com distribuição mundial, quase generalizada, podendo ser encontrados em desertos, savanas, cerrados, florestas tropicais, entre outros biomas, vivem frequentemente embaixo de folhas, pedras, madeiras, em fendas de rochas, entre tijolos ou quaisquer outros entulhos ou entre vegetações rasteiras e arbustos, habitam o planeta há milhões de anos, são encontrados em todos os continentes, com exceção da Antártida, sendo mais numerosos em ambientes áridos ou semiáridos. Em todo o mundo já foram registradas cerca de 2.239 espécies, com abrangência de 20 famílias e 165 gêneros (LOURENÇO; EICKSTEDT, 2009; AZEVEDO et al., 2017).

A fauna de escorpiões no Brasil ainda é subestimada pela carência de estudos específicos, porém até o momento, sabe-se da existência de 131 espécies, 23 gêneros e 4 famílias. As Famílias representadas são Buthidae, Bothriuridae, Chactidae e Liochelidae. Entre as regiões do país, o Nordeste se destaca por ser a segunda região mais rica em espécies escorpiônicas com 26\%, perdendo apenas para a região Norte que apresenta 52,0\% das espécies (PORTO et al., 2010a).

Em Sergipe é registrada a presença de 10 espécies pertencentes a duas Famílias de escorpiões: Buthidae, representada pelos gêneros, Tytius e Rhopalurus e a Família Bothriuridae, representada pelo gênero Bothriurus (ALMEIDA, 2010; PORTO et al., 2010b). Em relação aos acidentes escorpiônicos especificamente, Mesquita et al. (2015) avaliaram as informações obtidas das notificações dos casos de acidentes escorpiônicos ocorridos no Estado de Sergipe, através do sistema de informações do CIATOX no período entre janeiro de 2002 a dezembro de 2012, sugerindo que Sergipe tem uma elevada incidência de acidentes por escorpiões sendo, portanto, uma área endêmica, tendo necessidade de implantação de políticas públicas de saúde, preventivas deste agravo. 
No Brasil são quatro sistemas de notificação que registram dados acidentes por animais peçonhentos, o SINAN (Sistema de Informações de Agravos de Notificação), o SINITOX (Sistema Nacional de Informações Tóxico-Farmacológicas), o SIH-SUS (Sistema de Informações Hospitalares do Sistema Único de Saúde) e o SIM (Sistema de Informações sobre Mortalidade) (MESQUITA et al., 2015). Ademais o SINAN, seria o único sistema nacional que possui um módulo específico para tratar desse tipo de agravo à saúde e que contemplam o registro de acidentes por animais peçonhentos com o objetivo de gerar informações necessárias e suficientes para identificar o problema em estudos epidemiológicos (BOCHNER; STRUCHINER, 2002).

Identificar o envenenamento escorpiônico no estado de Sergipe pode apoiar a tomada de decisão na elaboração de atividades educativas interdisciplinares, tanto para a população em geral, para profissionais da saúde e de diferentes áreas. Logo, o presente estudo tem por objetivo identificar as características clínico-epidemiológicas dos casos de acidentes com escorpiões nos oito territórios do estado de Sergipe, bem como descrever os casos de óbito por picada e frequência de envenenamento escorpiônico, notificadas ao Sistema de Informação e de Agravos de Notificação - SINAN, no período entre 2004 a 2014.

\section{MATERIAL E MÉTODOS}

Estudo descritivo, retrospectivo utilizando uma abordagem quantitativa baseada em dados extraídos de relatórios de envenenamento envolvendo escorpiões venenosos nas doenças notificáveis. Sistema de Informação do SINAN no período entre 2004 a 2014.

O estudo foi realizado no estado de Sergipe, localizado no nordeste do Brasil, que ocupa uma área de 21.915,116 Km² e uma população estimada em 2018 de 2.278.308 habitantes (IBGE, 2019). A população é distribuída em oito territórios que formam a base para o planejamento de políticas públicas, e estas consistem em Alto Sertão, Médio Sertão, Baixo São Francisco, Agreste Centro, Leste, Grande Aracaju, Centro Sul e Sul (FALCON; SANDES, 2018).

Dados secundários foram coletados de registros de envenenamento escorpiônico no SINAN de 2004 a 2014 que foram obtidos do Estado Secretaria de Saúde de Sergipe.

Os dados foram estratificados de acordo com territórios de Sergipe. As seguintes variáveis foram analisadas: sexo (feminino e masculino), faixa etária, tempo decorrido entre o acidente e o atendimento médico, local anatômico da picada, classificação de gravidade, uso de soroterapia e evolução clínica das vítimas de acidentes escorpiônicos.

As frequências absolutas e relativas de acidentes escorpiônicos foram determinadas para cada território estudado. Além destas, o índice de letalidade dos acidentes escorpiônicos, no período entre 2004 a 2014, foram calculadas segundo Almeida-Filho e Barreto (2011), baseado nos dados populacionais fornecidos pelo IBGE nos anos base de 2007 e 2010 e na projeção populacional para os anos de 2004 a 2006, 2008, 2009 e 2011 a 2014 (IBGE, 2016).

Foram realizadas análises descritivas e análise de variância, a depender dos pré-requisitos e adequabilidade, utilizando-se ANOVA (One - Way) com Post Hoc do tipo Sidak ou o teste de Kruskal- 
-Wallis, todos com confiabilidade de 95,0\% $(P<0,05)$. Os cálculos estatísticos foram realizados por meio dos programas SPSS versão 21.0. e BioEstat versão 5.3.

O estudo foi aprovado pela pesquisa Comitê de Ética da Universidade de Tiradentes, com CAAE 39543814.3.0000.5371 e documento número 946982.

\section{RESULTADOS}

No que se refere a frequência dos acidentes escorpiônicos nos territórios de Sergipe verificou-se junto ao SINAN, que foram notificados, no período proposto para presente estudo, 8.021 casos de acidentes com animais peçonhentos no Estado de Sergipe, sendo que 5.133 (64,0\%), envolveram escorpiões, 80,0\% ocorreram na zona urbana, 9,5\% na zona rural e 4,0\% na zona periurbana.

Em relação a distribuição da população humana por sexo entre a zona rural e a zona urbana, em números absolutos, as mulheres em Sergipe são maioria na zona urbana (796.450/723.916) e os homens são maioria na zona rural (281.125/266.526). Os acidentes escorpiônicos que acontecem com homens e mulheres, distribuídos entre as zonas, nos territórios, a exceção do território da Grande Aracaju, ocorrem de forma semelhante (Tabela 1).

Tabela 1. Distribuição de acidentes escorpiônicos por sexo e território, no período entre 2004 e 2014, Sergipe.

\begin{tabular}{ccccc}
\hline Território & Feminino & Feminino \% & Masculino & Masculino \% \\
\hline Alto Sertão Sergipano & 15 & 44.1 & 19 & 55.9 \\
Médio Sertão Sergipano & 18 & 56.3 & 14 & 43.8 \\
Agreste Central Sergipano & 339 & 54.3 & 285 & 45.7 \\
Baixo São Francisco & 88 & 52.7 & 79 & 47.3 \\
Leste Sergipano & 39 & 50.6 & 38 & 49.4 \\
Grande Aracaju & 2304 & 58.5 & 1635 & 41.5 \\
Centro Sul Sergipano & 64 & 49.6 & 65 & 50.4 \\
Sul Sergipano & 65 & 49.6 & 66 & 50.4 \\
\hline
\end{tabular}

Fonte: Dados da pesquisa

$\mathrm{Na}$ análise da distribuição entre sexos das vítimas de acidentes escorpiônicos entre todos os territórios, observou-se que o sexo feminino foi o mais acometido por acidentes escorpiônicos em Sergipe $(57,1 \%)$, com diferença estatisticamente significativa entre os territórios $(p=0,025)$. 0 território da Grande Aracaju foi o único que demonstrou diferenças estatisticamente significativa entre acidentes acometendo homens e mulheres na zona urbana e rural (p0,05). 
Ao analisar a distribuição de casos de acidentes escorpiônicos entre as faixas etárias, se encontrou maiores proporções entre as faixas de 20 a 24 anos (10,3\%) e 25 a 29 anos (9,3\%). No comparativo entre as faixas etárias por território, foram encontradas diferenças significativas (p0,01).

Quanto a distribuição dos acidentes por faixa etária nos territórios, destacaram-se o Médio Sertão na faixa entre 15 a 19 (18,8\%); o Agreste (10,9\%), no Baixo São Francisco (11,4\%) e na Grande Aracaju (10,3\%) a faixa etária de 20 a 24 foi a mais acometida. No Centro Sul de 25 a 29 anos foi a faixa mais atingida (18,6\%). No Leste a faixa etária de 30 a 34 foi a mais acometida (14,3\%). No Alto Sertão a faixa etária mais acometida foi a de 35 a 39 (29,4\%). No Sul, as faixas etárias de 40 a 44 e 45 a 49 anos foram as mais acometidas $(9,2 \%)$.

Ocorreram no período entre 2004 até 2014 quatro óbitos, sendo que a taxa de letalidade calculada nesse estudo para o período foi de 0,076 (4/5.133). Em relação a distribuição por zona, três ocorreram em zona urbana e um em zona rural. A distribuição dos óbitos por zona, acompanha a distribuição da ocorrência de acidentes, considerando que mais de $80,0 \%$ dos acidentes escorpiônicos ocorreram em zona urbana e o território da Grande Aracaju, concentrou mais de 75,0\% dos casos em Sergipe, no período estudado.

Quanto ao perfil das vítimas, duas foram do sexo masculino e duas do sexo feminino, a faixa etária predominante foi entre 0 e 9 anos ( 2 casos), um adolescente entre 15 e 19 anos e um idoso entre 65 e 69 anos. Todos os atingidos buscaram atendimento em até 3 horas. Quanto à anatomia atingida das vítimas, três foram atingidos em membros superiores e em um deles, não foi registrado o local anatômico do acidente.

Dos quatro casos de óbito, apenas um recebeu tratamento soroterápico, sendo que o mesmo recebeu doses superiores as que são preconizadas no protocolo do Ministério da Saúde (MS). Dos quatro casos de óbito, apenas um recebeu tratamento soroterápico, sendo que o mesmo recebeu doses superiores as que são preconizadas no protocolo do MS.

Quanto aos dados clínico-epidemiológicos relacionados aos acidentes escorpiônicos constatou-se que as vítimas de acidentes escorpiônicos registradas em Sergipe, tiveram como localização anatômica do corpo mais atingida, membros inferiores com $37,1 \%$, seguido dos membros superiores com frequência 36,0\%. Além das áreas anatômicas do corpo mais atingidas, foram distribuídas as demais áreas de acordo com os territórios de Sergipe (Tabela 2), período entre 2004 a 2014.

Tabela 2. Distribuição anatômica dos acidentes escorpiônicos registrados nos territórios de Sergipe entre 2004 e 2014.

\begin{tabular}{cccccccc} 
Território & Frequências & $\begin{array}{c}\text { Membros } \\
\text { superiores }\end{array}$ & Cabeça & Tronco & $\begin{array}{c}\text { Membros } \\
\text { Inferiores }\end{array}$ & Ignorado & Total \\
\hline ALTO & Absoluta & 19 & 2 & 1 & 11 & 1 & 34 \\
SERTÃO & Relativa & $55.90 \%$ & $5.90 \%$ & $2.90 \%$ & $32.40 \%$ & $2.90 \%$ & $100 \%$ \\
\hline MEDIO & Absoluta & 10 & 0 & 1 & 10 & 11 & 32 \\
SERTÃO & Relativa & $31.30 \%$ & $0.00 \%$ & $3.10 \%$ & $31.30 \%$ & $34.40 \%$ & $100 \%$ \\
\hline
\end{tabular}




\begin{tabular}{cccccccc}
\hline Território & Frequências & $\begin{array}{c}\text { Membros } \\
\text { superiores }\end{array}$ & Cabeça & Tronco & $\begin{array}{c}\text { Membros } \\
\text { Inferiores }\end{array}$ & Ignorado & Total \\
\hline AGRESTE & Absoluta & 199 & 12 & 20 & 201 & 192 & 624 \\
CENTRAL & Relativa & $31.90 \%$ & $1.90 \%$ & $3.20 \%$ & $32.20 \%$ & $30.8 \%$ & $100 \%$ \\
BAIXO SÃO & Absoluta & 83 & 2 & 6 & 65 & 11 & 167 \\
FRANCISO & Relativa & $49.70 \%$ & $1.20 \%$ & $3.60 \%$ & $38.90 \%$ & $6.60 \%$ & $100 \%$ \\
& Absoluta & 29 & 0 & 2 & 26 & 20 & 77 \\
LESTE & Relativa & $37.7 \%$ & $0.00 \%$ & $2.60 \%$ & $33.80 \%$ & $26.0 \%$ & $100 \%$ \\
GRANDE & Absoluta & 1385 & 67 & 156 & 1486 & 845 & 3939 \\
ARACAJU & Relativa & $35.20 \%$ & $1.70 \%$ & $4.00 \%$ & $37.70 \%$ & $21.5 \%$ & $100 \%$ \\
CENTRO & Absoluta & 60 & 2 & 4 & 56 & 7 & 129 \\
SUL & Relativa & $46.50 \%$ & $1.60 \%$ & $3.10 \%$ & $43.40 \%$ & $5.40 \%$ & $100 \%$ \\
& Absoluta & 62 & 4 & 3 & 51 & 11 & 131 \\
SUL & Relativa & $47.30 \%$ & $3.10 \%$ & $2.30 \%$ & $38.90 \%$ & $8.40 \%$ & $100 \%$ \\
\hline
\end{tabular}

Fonte: Dados da pesquisa

Quanto a classificação de gravidade dos casos de acidentes com escorpiões em Sergipe, no período proposto por esse estudo, encontrou-se que a maior parte dos casos foram diagnosticados clinicamente como leves (84,4\%); vale ressaltar o percentual elevado de casos não registrados ou ignorados, quanto a gravidade ou classificação (10.50\%). A distribuição da classificação de gravidade estratificada por território é apresentada na Tabela 3. Em que se observou diferenças estatisticamente significativas entre os territórios em relação a essas classificações (p0,0001).

Tabela 3. Distribuição da classificação de gravidade dos acidentes escorpiônicos nos territórios de Sergipe, no período entre 2004 e 2014.

\begin{tabular}{ccccccc}
\hline Território & Frequências & Leve & Moderado & Grave & Ignorado & Total \\
\hline \multirow{2}{*}{ ALTO SERTÃO } & Absoluta & 21 & 10 & 0 & 3 & 34 \\
& Relativa & $61.80 \%$ & $29.40 \%$ & $0.00 \%$ & $8.80 \%$ & $100 \%$ \\
& Absoluta & 22 & 2 & 1 & 7 & 32 \\
MEDIO SERTÃO & Relativa & $68.80 \%$ & $6.30 \%$ & $3.10 \%$ & $21.90 \%$ & $100 \%$ \\
\hline \multirow{2}{*}{ AGRESTE } & Absoluta & 424 & 27 & 0 & 173 & 624 \\
CENTRAL & Relativa & $67.90 \%$ & $4.30 \%$ & $0.00 \%$ & $27.70 \%$ & $100 \%$ \\
\hline
\end{tabular}




\begin{tabular}{ccccccc}
\hline Território & Frequências & Leve & Moderado & Grave & Ignorado & Total \\
\hline BAIXO SÃO & Absoluta & 148 & 11 & 2 & 6 & 167 \\
FRANCISO & Relativa & $88.60 \%$ & $6.60 \%$ & $1.20 \%$ & $3.60 \%$ & $100 \%$ \\
& Absoluta & 66 & 6 & 0 & 5 & 77 \\
LESTE & Relativa & $85.70 \%$ & $7.80 \%$ & $0.00 \%$ & $6.50 \%$ & $100 \%$ \\
& Absoluta & 3447 & 174 & 12 & 306 & 3939 \\
GRANDE ARA- & Relativa & $87.50 \%$ & $4.40 \%$ & $0.30 \%$ & $7.80 \%$ & $100 \%$ \\
CAJU & Absoluta & 108 & 10 & 0 & 11 & 129 \\
& Relativa & $83.70 \%$ & $7.80 \%$ & $0.00 \%$ & $8.50 \%$ & $100 \%$ \\
SUIRO SUL & Absoluta & 94 & 8 & 1 & 28 & 131 \\
& Relativa & $71.80 \%$ & $6.10 \%$ & $0.80 \%$ & $21.40 \%$ & $100 \%$ \\
\hline
\end{tabular}

Fonte: Dados da pesquisa

Em Sergipe, 14,26\% das 5.133 pessoas que sofreram acidentes escorpiônicos, receberam soroterapia no tratamento dos sintomas (Tabela 4), desses conforme a classificação registrada, nem todos teriam a indicação de uso, outros utilizaram soroterapia sem o registro da classificação $(9,8 \%)$ e em $6,0 \%$ dos casos não se sabe a classificação e se utilizaram ou não a soroterapia.

Tabela 4. O uso de soroterapia relacionado com a classificação de gravidade dos acidentes escorpiônicos no Estado de Sergipe, no período entre 2004 e 2014.

\begin{tabular}{cccccc}
\hline \multirow{2}{*}{ Classificação } & & \multicolumn{3}{c}{ Soroterapia } & \multirow{2}{*}{ TOTAL } \\
\cline { 3 - 5 } & & Sim & Não & Ignorado & \\
\hline \multirow{2}{*}{ Leve } & Absoluta & 481 & 163 & 4330 & 4330 \\
& Relativa & $11,1 \%$ & $3,8 \%$ & $85,1 \%$ & $100 \%$ \\
\multirow{2}{*}{ Moderado } & Absoluta & 185 & 49 & 14 & 248 \\
& Relativa & $74,6 \%$ & $19,8 \%$ & $5,6 \%$ & $100 \%$ \\
\cline { 3 - 5 } Ignove & Absoluta & 13 & 1 & 2 & 16 \\
& Relativa & $81,3 \%$ & $6,3 \%$ & $12,5 \%$ & $100 \%$ \\
\hline & Absoluta & 53 & 179 & 307 & 539 \\
& Relativa & $9,8 \%$ & $33,2 \%$ & $57 \%$ & $100 \%$ \\
\hline
\end{tabular}

Fonte: Dados da pesquisa 
Na busca pelo atendimento médico após os acidentes escorpiônicos em Sergipe, 56,0 \% das vítimas buscaram o atendimento em até 3 horas e casos ignorados ou não registrados 28,9\%. Quanto aos territórios em relação ao tempo de procura por atendimento médico, constatou-se que existem diferenças estatisticamente significativas entre os mesmos (p0,0001) (Tabela 5).

Tabela 5. 0 tempo decorrido do momento dos acidentes escorpiônicos, até o atendimento médico, apresentado por Territórios do Estado de Sergipe, no período entre 2004 e 2014.

\begin{tabular}{ccccc}
\hline \multirow{2}{*}{ Território } & Frequências & Até 3 horas & Mais de 3 horas & Total \\
\hline \multirow{2}{*}{ ALTO SERTÃO } & Absoluta & 28 & 6 & 34 \\
& Relativa & $82,3 \%$ & $17,7 \%$ & $100 \%$ \\
MEDIO SERTÃO & Absoluta & 15 & 17 & 32 \\
& Relativa & $46,9 \%$ & $53,1 \%$ & $100 \%$ \\
AGRESTE CENTRAL & Absoluta & 279 & 345 & 624 \\
\multirow{2}{*}{ BAIXO SÃO FRANCISO } & Relativa & $44,7 \%$ & $53,3 \%$ & $100 \%$ \\
& Absoluta & 114 & 53 & 167 \\
& Relativa & $68,2 \%$ & $31,8 \%$ & $100 \%$ \\
LESTE & Absoluta & 36 & 41 & 77 \\
& Relativa & $46,8 \%$ & $53,2 \%$ & $100 \%$ \\
GRANDE ARACAJU & Absoluta & 2240 & 1699 & 3939 \\
& Relativa & $56,8 \%$ & $43,2 \%$ & $100 \%$ \\
CENTRO SUL & Absoluta & 81 & 48 & 129 \\
& Relativa & $62,8 \%$ & $37,2 \%$ & $100 \%$ \\
& Absoluta & 77 & 54 & 131 \\
\hline SUL & Relativa & $58,8 \%$ & $41,2 \%$ & $100 \%$ \\
\hline
\end{tabular}

Fonte: Dados da pesquisa

Procurando encontrar as diferenças de sintomatologia entre os territórios, se calculou as frequências absolutas e relativas em cada território (Tabela 6). As manifestações locais mais frequentes nos casos de acidentes escorpiônicos em Sergipe, no período estudado foram dor, edema e eritema. Além disso, as manifestações sistêmicas mais frequentes foram as vagais, hemorrágicas e miolíticas. Entre os territórios, em relação as referidas manifestações, foram encontradas diferenças estatísticas significativas $(\mathrm{p} 0,05)$. 


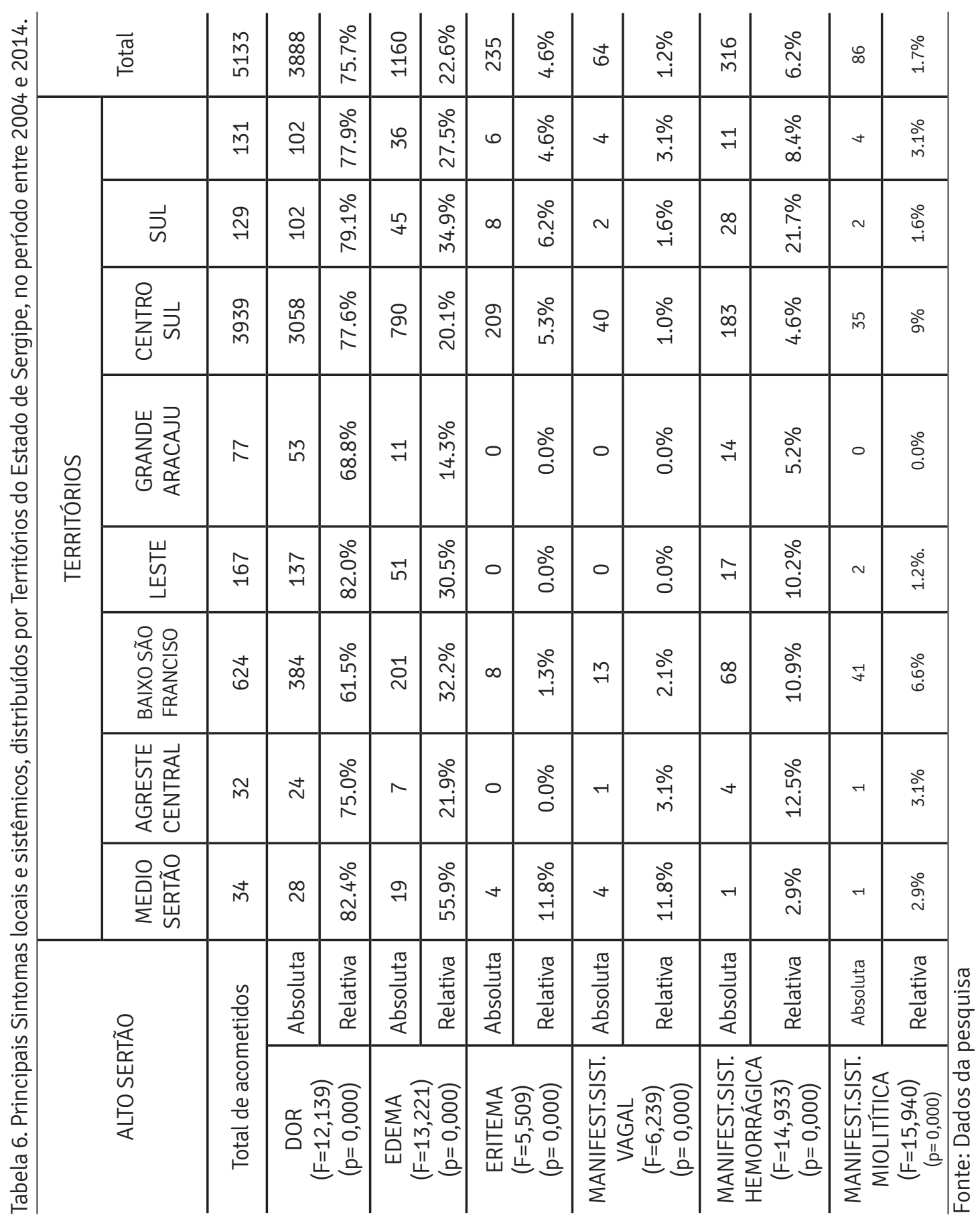


Em relação a manifestação local "DOR” o território do Alto Sertão apresentou a maior frequência relativa, contudo não apresentou diferenças estatísticas significativas dos demais territórios $(p 0,05)$, o território do Agreste Central apresentou a menor frequência relativa de ocorrência de dor entre os territórios, com diferenças estatísticas significativas dos territórios do Baixo São Francisco, Grande Aracaju, Centro Sul e Sul sergipanos.

Nas manifestações locais com Edema, o território do Alto Sertão apresentou a maior frequência relativa entre os territórios, com diferenças estatísticas significativas dos demais territórios (p0,05), com exceção ao território Centro Sul, que nesse caso, não demonstra diferenças estatísticas do Alto Sertão.

Nas manifestações locais do tipo Eritema, a maior frequência relativa foi no território do Alto Sertão, contudo não se encontrou diferenças estatísticas significativas dos demais territórios (p0,05), 0 território da Grande Aracaju, ao contrário, demonstrou diferenças estatísticas quanto a manifestação Eritema, dos territórios do Agreste Central e Baixo São Francisco.

Quanto as manifestações sistêmicas vagais, o território do Alto Sertão apresentou a maior frequência relativa com diferenças estatísticas significativas dos demais territórios (p0,05). 0 território Centro Sul foi o que apresentou a maior frequência relativa de manifestações sistêmicas hemorrágicas entre os territórios, com diferenças estatísticas significativas dos demais, a exceção do Médio Sertão $(\mathrm{p0}, 05)$. As manifestações miolíticas tiveram a maior frequência relativa no território do Agreste Central, com diferenças estatísticas significativas dos territórios Baixo São Francisco, Leste, Grande Aracaju, Centro Sul e Sul (p0,05).

\section{DISCUSSÃO}

No presente estudo o sexo feminino foi o mais acometido com os acidentes escorpionicos, principalmente no território da Grande Aracaju; em diversos outros estudos sobre acidentes escorpiônicos, as vítimas do sexo feminino foram as mais acometidas, são exemplos os achados de Mesquita et al. (2015) e Barros et al. (2014). Para Albuquerque et al. (2004) a maior frequência de acidentes escorpiônicos ocorreram entre mulheres devido a essas realizarem no ambiente doméstico atividades de limpeza em locais habitados por escorpiões estando esse gênero mais vulnerável aos acidentes.

No presente estudo, em relação ao sexo das vítimas se apresenta para a maior parte dos territórios, com resultados semelhantes aos encontrados por Reckziegel e Pinto (2014), que em seu estudo discutem sobre a diferença nas proporções de acidentes entre homens e mulheres ser pequena e atribuem a causa a uma provável semelhança de riscos entre os dois sexos, baseados nas características da vida urbana e doméstica.

No Brasil foram identificados 691.147 casos de acidentes por animais peçonhentos notificados em que $40,0 \%$ referiu-se a acidentes com escorpiões. A Região Nordeste responsável pela maioria das notificações $(47,0 \%)$, seguida da Sudeste $(41,0 \%)$. Em relação aos gêneros foram igualmente acometidos, 50,0\% homens e 50,0\% mulheres, entre 20-39 anos. A grande maioria das vítimas evoluiu à cura (93,0\%), tendo sido realizado o atendimento entre 0 -1 hora na maior parte das vezes (47,0\%) (AMARAL et al., 2016). 
Em relação as faixas etárias mais acometidas, ocorreram com variações entre os territórios e os dados encontrados concordam com os reportados por outros autores que afirmam que as faixas etárias mais acometidas coincidem com as faixas etárias das pessoas economicamente ativas (QUADROS et al., 2014; BARROS et al., 2014). Essas informações coincidem com os achados de Mesquita et al. (2015) que apontaram como faixa etária mais acometida em Sergipe as idades entre 20 e 29 anos.

No presente estudo ocorreram quatro óbitos no estado de Sergipe, sendo que a taxa de letalidade calculada nesse estudo para o período foi de 0,076 (4/5.133). Esse dado diverge de Mesquita et al. (2015), que encontrou no período entre os anos de 2002 e 2012, dois óbitos e uma taxa de letalidade de 0,06 (2/3.547). Essa diferença está relacionada a variação de período e as diferenças de sensibilidade e captação dos sistemas de informação, já que na pesquisa citada foram utilizados dados secundários do SINITOX e o presente trabalho utilizou o SINAN como fonte de dados.

Segundo dados do Ministério da Saúde, em 2011 ocorreram 137.421 acidentes por animais peçonhentos no Brasil, sendo que 51 pacientes evoluíram para o óbito. Além disso esses óbitos acometeram principalmente trabalhadores do campo e florestas, nas regiões Norte, Nordeste e Centro-Oeste do território nacional, que podem estar relacionados a idade da vítima, a quantidade de veneno inoculado e o tempo decorrido entre a picada e o acesso ao tratamento adequado (CHIPPAUX, 2015).

Existiu um equilíbrio de acometimento quanto ao sexo, nos casos de óbitos. No caso da idade e o tempo de busca ao atendimento médico após os acidentes, essas variáveis têm influência direta na qualidade do prognóstico, sendo que crianças de até 10 anos tem maior risco para gravidade dos casos (CUPO, 2015). Houve uma tendência de maior acometimento dos casos de óbitos em crianças e essa pode ter ocorrido, devido ao fato que muitas vezes essas brincarem ao chão, aumentando as chances de serem picadas (ALBUQUERQUE et. al., 2013).

Quando se analisa os resultados sobre membros anatômicos mais atingidos, ao verificar os dados de Sergipe pode-se concluir que os membros superiores e inferiores são acometidos de forma semelhante, porém ao estratifica-se por território, é possível detectar as diferenças desse perfil em determinados locais, como por exemplo o território do Alto Sertão. Em função da baixa densidade de acidentes e da deficiência nas informações constantes no sistema sobre a relação entre acidentes e o trabalho, não será possível, nesse estudo elucidar tal diferença, sendo necessários outros trabalhos para identificar tal informação.

Apesar do aparente equilíbrio no acometimento de membros superiores e inferiores, chama atenção o índice de registros ignorados nessa variável, considerando que a falta dessa informação inviabiliza o conhecimento do perfil em relação aos membros mais atingidos e prejudica o estabelecimento de ações preventivas mais específicas. Observou-se que o território do Alto Sertão apresentou a maior parte dos casos atingindo membros superiores, seguido do Baixo São Francisco e do Sul sergipanos. Segundo Ribeiro et al. (2001), os escorpiões tendem a picar pessoas em membros superiores quando, inadvertidamente manipulam objetos ou entulhos sem a devida proteção ou os devidos cuidados.

As ações de prevenção deverão ser direcionadas aos grupos mais vulneráveis para esse tipo de acidente e, além disso a localização dos acidentes influencia no prognóstico de gravidade do caso. A anatomia atingida influencia na gravidade e quanto mais próximo de órgãos vitais for o acometi- 
mento, maiores poderão ser as complicações e sequelas do acidente (PARDAL et al., 2014). Os escorpiões normalmente atingem os pés das pessoas no ato de calçar sapatos e as mãos, no momento da manipulação de materiais e entulhos (RIBEIRO et al., 2001).

Chama a atenção os casos de acidentes escorpiônicos com classificação moderada, em que 19,8\% não utilizaram soro, da mesma forma que $6,3 \%$ dos casos graves, deveria utilizar a soroterapia e o sistema de informações não declara o uso. Se indicou a soroterapia indevidamente, por outro lado, entre os casos de classificação de gravidade ignorada, $57,0 \%$ não foram registradas quanto ao uso ou não de soroterapia. Fiszon e Bochner (2008), identificou que as dificuldades em notificar SINAN podem ser parcialmente explicados pela baixa valorização dada no passado à informação por parte das instituições e profissionais de saúde, bem como nos casos caso dos acidentes por animais peçonhentos.

De acordo com Salomão et al. (2018), nem todas as fichas de notificação são disponibilizadas ao SINAN, além disso a ausência de acesso amplo a esses dados impede que importantes indicadores sejam considerados em estudos epidemiológicos. Ainda assim a associações entre tipo de acidente, sintomatologia, soro utilizado ou gravidade do acidente e número de ampolas aplicadas, não são verificáveis pela consulta ao site, dificultando a eficiência de tratamento, qualidade de diagnóstico e preenchimento dos dados. Além disso a falta de sincronia e interligação dos Sistemas de informação nas esferas federal, estadual e municipal, resultam em discrepâncias na apresentação e análise dos dados (MACHADO; BOCHNER, 2012).

De acordo com o Ministério da Saúde (BRASIL, 2009) nos casos moderados, deve-se indicar duas a três ampolas de soroterapia antiescorpiônica e nos casos graves, recomenda-se a aplicação de quatro a seis ampolas, Cupo (2015) recomenda o uso de 3 ampolas para os casos moderados e 6 ampolas para os casos graves, recomendando ainda a aplicação de soroterapia, independente do quadro de gravidade para todas as crianças de 0 a 7 anos que forem atingidas.

Conforme Ribeiro e Melo (2012) o veneno dos escorpiões apresenta diversas toxinas de composição proteica com baixa quantidade de neurotoxinas, a ação ocorre nos canais de sódio e potássio, influenciando na estimulação de nervos periféricos sensitivos, motores e do sistema nervoso autônomo (RIBEIRO et al., 2009), portanto a dor e o eritema são achados frequentes nos acidentes com escorpiões, pois com a inoculação de alguma substância estranha ao organismos, imediatamente ocorrem a migração de células de defesa para o local da picada e o aumenta a perfusão sanguínea local, com esse aumento, ocorrem o edema, eritema e dor, que traduzem os sinais inflamatórios (PARDAL et. al., 2014).

Quanto a gravidade dos casos, a maior parte enquadrou-se como leves, achados semelhantes foram encontrados por Mesquita et al. (2015). Conforme Cupo (2015) a maior parte dos acidentes escorpiônicos, em torno de 85,0 a 90,0\% vão apresentar apenas sintomas locais leves, como dor no local da picada e parestesia. Nos casos moderados, outros sintomas foram relatados, em especial os sintomas decorrentes de manifestações vagais, tais como náuseas e vômitos. Já os casos graves, sinais de depressão do sistema nervoso central, como prostração, bradicardia, insuficiência cardíaca e edema pulmonar podem estar presentes

Conforme o protocolo do Ministério da Saúde do Brasil, o soro antiescorpiônico é indicado em todos os casos classificados como moderado ou grave, já ao contrário nos casos Leves, não existe 
a indicação de soroterapia, a exceção de crianças menores de 7 anos, quando se indica o uso, de forma profilática (BRASIL, 2009).

A distribuição de soros é realizada de acordo com as características regionais da ocorrência dos acidentes notificados pelo SINAN, daí a importância de se registrar esse agravo. Apesar disso, sabe-se que a subnotificação ainda é um desafio a ser superado pelo SUS, o que pode impactar diretamente no subdimensionamento de distribuição dos soros no país (SALOMÃO et al., 2018). 0 sub-registro e a subnotificação podem ser piores quando se trata de trabalhadores rurais, regiões onde podem existir situações de precariedade dos serviços de saúde ou onde os profissionais de saúde têm dificuldade de estabelecer a relação com o trabalho (PARDAL et al., 2004).

Observa-se que o território do Alto Sertão é o que proporcionalmente, busca atendimento mais rápido, em relação aos demais territórios, enquanto o Agreste Central, proporcionalmente é o que mais demora para a busca de atendimento. 0 território do Alto Sertão apresentou uma baixa densidade de acidentes nos 11 anos estudados, porém esse se destaca porque demonstra que os casos que se tem registro no período, ao que se percebe, têm os maiores percentuais de casos moderados do Estado e fez com que as vítimas buscassem de forma mais imediata o atendimento. Contudo, essa afirmativa não é conclusa, pois, sabe-se que em muitos casos, pessoas são picadas e não manifestam reações importantes, deixando de buscar atendimento e registrar o acidente

Quanto aos sintomas hemorrágicos, estão sempre descritos no caso de acidentes com serpentes. Os escorpiões têm em seu veneno propriedades que não interferem, em princípio das atividades hemolíticas (CUPO et al., 2009) ou hemorrágicas, no entanto por estímulo parassimpático podem ocorrer vasodilatação das artérias e arteríolas, e uma pessoa com alterações sistêmicas anteriores poderia apresentar hemorragia, secundária ao acidente.

\section{CONCLUSÃO}

Nos territórios de Sergipe verificou-se junto ao SINAN, que foram notificados, no período proposto, 5.133 casos de acidentes por escorpião. Destes, ocorreram quatro óbitos no período estudado, sendo 75,0\% deles em crianças e adolescentes; a faixa etária mais acometida em Sergipe foi entre 20 e 29 anos e constatou-se que o sexo feminino foi o mais atingido em função das atividades domésticas e do aspecto da sinantropia, aumentando o risco, especialmente para as mulheres que ficam mais expostas aos escorpiões.

Quanto aos dados clínico-epidemiológicos tiveram como localização anatômica do corpo mais atingida, membros inferiores e membros superiores. Já na classificação de gravidade dos casos, a maioria foram diagnosticados clinicamente como leves $84,4 \%$. Em relação a busca pelo atendimento médico após os acidentes escorpiônicos, 56,0 \% das vítimas buscaram o atendimento em até 3 horas. As manifestações locais mais frequentes nos casos de acidentes no período estudado foram dor, edema e eritema. Além disso, as manifestações sistêmicas mais frequentes foram as vagais, hemorrágicas e miolíticas. 
Conhecer os territórios escorpiônicos é de fundamental importância para que nesses locais se redobre os cuidados quanto aos hábitos de manter o ambiente doméstico livre de entulhos, evitar possíveis cavidades nas estruturas de paredes das casas, utilizar telas nos esgotos e encanamentos e utilizar equipamentos de proteção individual, evitando ao máximo a invasão e destruição dos predadores naturais dos escorpiões e dos espaços naturais, que ainda se preservam.

Este estudo, por tratar-se de análise de banco de dados do SINAN e está sujeito a erros durante a inserção das informações e à subnotificação, no entanto, por chamar a atenção da elevada incidência do escorpionismo em Sergipe, se fez necessário esta pesquisa. Além disso, sugere a realização de estudos que permitam a análise mais específica da incidência e informações necessárias, suficientes para se conhecer a dimensão real dessa situação epidemiológica.

Educação da população e profissionais de diversos setores, especialmente o setor da saúde, sobre o impacto na promoção da saúde e prevenção de acidentes com animais venenosos é importante para uma melhor proteção das pessoas e meio ambiente. Ademais, o incentivo e capacitação aos profissionais da saúde responsáveis pelo preenchimento e notificação dos dados, bem como a demonstração da real importância destes dados podem melhorar a comunicação entre a esfera federal, estadual e municipal, sendo possível melhores intervenções de acordo com as necessidades de cada área do território nacional.

\section{REFERÊNCIAS}

ALBUQUERQUE, C.M.R. et al. Pediatric epidemiogical aspects of scorpionism and report on fatal cases from Tytius stigmurus stings (Scorpiones: Buthidae) in State of Pernanbuco, Brasil. Rev Soc Bras Med Trop, v. 46, n. 4, p. 484-489, 2013.

ALBUQUERQUE, I.C.S. et al. Escorpionismo em Campina Grande (PB). Rev Biol Cienc Terra, v. 4, n. 1, p. 2-10, 2004.

ALMEIDA FILHO, N.; BARETTO M.L. Epidemiologia e saúde: fundamentos, métodos e aplicações. Rio de Janeiro: Guanabara Koogan, 2011.

ALMEIDA, R.B. Atlas das espécies de Tytius, CL Koch, 1836 (Scorpiones, Buthidae) do Brasil. 2010. 154f. Dissertação (Mestrado) - Universidade de São Paulo, São Paulo, 2010.

AMARAL, N.A.C et al. Análise dos casos de acidentes escorpiônicos no Brasil. Rev Saúde, v.10, n.1, p. 114, 2016.

AZEVEDO, R. et al. Acidentes causados por aranhas e escorpiões no Estado do Ceará, Nordeste do Brasil: casos subnotificados e superestimados baseados na distribuição geográfica das espécies.

Pesq Ens Ciênc Exatas Nat, v. 1, n. 2, p. 144-158, 2017. 
BARROS, R.M. et al. Clinical and epidemiological aspects of scorpion stings in the northeast region of Brazil. Cien Saúde Col, v. 19, n. 4, p. 1275-1282, 2014.

BOCHNER, R.; STRUCHINER, C.J. Acidentes por animais peçonhentos e sistemas nacionais de informação. Cad Saúde Pública Rio de Janeiro, v.18, n.3, p.735-746, 2002.

BRASIL. Ministério da Saúde. Manual de controle de escorpiões. Série B. Textos Básicos de Saúde. Brasília: MS, 2009.

CHIPPAUX, J.P. Epidemiology of envenomations by terrestrial venomous animals in Brazil based on case reporting: from obvious facts to contingencies. J Venom Anim Toxins Incl Trop Dis, v. 21, n. 1, p. 13-17, 2015.

CUPO, P. Clinical Update on Scorpion Envenoming. Rev Soc Bras Med Trop, v. 48, n. 6, p. 642-649, 2015.

CUPO, P. et al. Escorpiões de importância médica. In: CARDOSO, J.L.C. et al. Animais peçonhentos no Brasil: biologia clínica e terapêutica dos acidentes. São Paulo: Salvier, 2009.

FALCON, M.L.O.; SANDES, M.A.S. Desenvolvimento, Territorialidade e Cultura: a experiência de Sergipe em planejamento regional nos anos 2007-2013. RBPO, v. 8, n. 1, p. 45-56, 2018.

FISZON, J.T.; BOCHNER, R. Subnotificação de acidentes por animais peçonhentos registrados pelo SINAN no Estado do Rio de Janeiro no período de 2001 a

2005. Rev Bras Epidemiol. v. 11, n. 1, p. 114-27, 2008.

IBGE. Instituto Brasileiro de Geografia e Estatística. Estimativa da população (2004, 2005, 2006, 2008, 2009, 2011, 2012, 2013 e 2014). 2016. Disponível em:<https://www.ibge.gov.br/estatisticas/ sociais/populacao/9103-estimativapopulacao.html?=\&t=o-que-e>. Acesso em: 08 set 2016.

IBGE. Instituto Brasileiro de Geografia e Estatística. Estimativa da população (2018). 2018. Disponível em:<https://www.ibge.gov.br/estatisticas/sociais/populacao/9103-estimativapopulacao. html?=\&t=o-que-e>. Acesso em: 16 jul 2019.

LOURENÇO, W.R.; EICKSTEDT, V.R. Escorpiões de importância médica. In: CARDOSO, J.L.C. et al. Animais peçonhentos no Brasil: Biologia, clínica e terapêutica dos acidentes. v. 2. São Paulo: Sarvier , 2009.

MACHADO, C; BOCHNER, R. A informação dos acidentes crotálicos no Estado do Rio de janeiro, 2001 a 2010. Gaz Med Bahia, v. 82, n. 1, p. 78-84, 2012. 
MESQUITA, F. et al. Acidentes escorpiônicos no Estado de Sergipe - Brasil. Rev Fac Cien Med Sorocaba, v. 17, n. 1, p. 15-20, 2015.

PARDAL, P.P.O. et al. Clinical trial of two antivenoms for the treatment of Bothrops and Lachesis bites in the north eastern Amazon region of Brazil. Trans R Soc Trop Med Hyg, v. 98, n. 1, p. 28-42, 2004.

PARDAL, P.P.O. et al. Envenenamento grave pelo escorpião Tytius obscurus Gervais, 1843. Rev PanAmaz Saude, v. 5, n. 3, p. 65-70, 2014.

PORTO, T.J. et al. Diversidade de escorpiões no Brasil. In: BRAZIL, T.K.; PORTO, T. Os Escorpiões. Salvador: EDUFBA. 2010a.

PORTO, T.J. et al. Primeiro relato de acidentes escorpiônicos causados por Tytius martinpaechi Lourenço, 2001 (Scorpiones; Buthidae). Rev Cienc Med Biol, v. 9, n. 3, p. 266-269, 2010 b.

QUADROS, R.M. et al. Acidentes escorpiônicos notificados pelo SINAN na Região Serrana de Santa Catarina, Brasil, 2000 - 2010. Rev Eletron Biol, v. 7, n. 1, p. 96-108, 2014.

RECKZIEGEL, G.C.; PINTO JUNIOR, V.L. Análise do escorpionismo no Brasil no período de 2000 a 2010. Rev Pan-Amaz Saude, v. 5, n. 1, p. 67-68, 2014.

RIBEIRO, E.L. et al. Hemograma de cães submetidos ao envenenamento experimental por Tytius serrulatus. Arq Bras Med Vet Zootec, v. 61, n. 1, p. 135-143, 2009.

RIBEIRO, E.L.; MELO, M.M. Proteínas do soro sanguíneo de cães inoculados com veneno de Tityus serrulatus. Arq Bras Med Vet Zootec, V. 64, n.1, p. 217-220, 2012.

RIBEIRO, L.A. et al. Aspectos clínicos e epidemiológicos do envenenamento por escorpiões em São Paulo e municípios próximos. Rev Patol Trop, v. 30, n. 1, p. 83-92, 2001.

SALOMÃO, M.G et al. Epidemiologia dos acidentes por animais peçonhentos e a distribuição de soros: estado de arte e a situação mundial. Rev Saúde Públ, v. 20, n. 4, p. 523-529, 2018. 
Recebido em: 3 de Abril de 2019

Avaliado em: 12 de Julho de 2019

Aceito em: 12 de Julho de 2019

2 Enfermeiro pela Universidade Federal do Rio Grande do Sul; Mestre pelo Programa de Pós-graduação em Saúde e Ambiente, Universidade Tiradentes, Aracaju, SE. E-mail: adriano.aracaju@gmail.com

3 Enfermeiro pela Universidade Tiradentes, SE; Doutorando do Programa de Pós-graduação em Saúde e Ambiente, Universidade Tiradentes, Aracaju, SE. E-mail: guilhermemota.enf@gmail.com

4 Doutorado em Ecologia e Recursos Naturais Universidade Estadual do Norte Fluminense Darcy Ribeiro; Docente do Programa de Pós-Graduação em Saúde e Ambiente, Universidade Tiradentes, Aracaju, SE. ORCID: 0000.0001.9042.682X. E-mail: andscoelho@yahoo.com

\section{唯

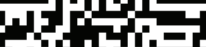

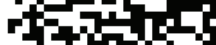

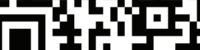

A autenticidade desse artigo pode ser conferida no site https://periodicos. set.edu.br

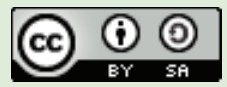

Este artigo é licenciado na modalidade acesso abertosob a Atribuição-Compartilhalgual CC BY-SA

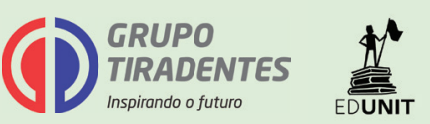

